\title{
The Novel PrisMax Continuous Renal Replacement Therapy System in a Multinational, Multicentre Pilot Setting
}

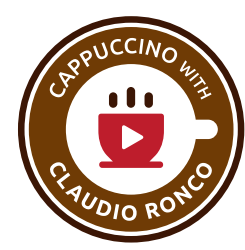

\author{
Marcus Broman ${ }^{a}$ Max Bell ${ }^{b, c}$ Olivier Joannes-Boyaud ${ }^{d}$ Claudio Ronco ${ }^{e, f}$ \\ ${ }^{a}$ Department of Perioperative and Intensive Care, Skåne University Hospital, Lund, Sweden; ${ }^{b}$ Department of \\ Perioperative Medicine and Intensive Care, Karolinska University Hospital, Stockholm, Sweden; ' ${ }^{\circ}$ Department

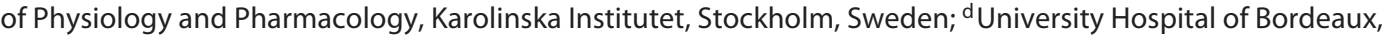

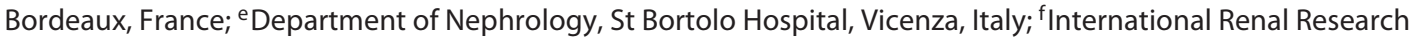 \\ Institute Vicenza, Vicenza, Italy
}

\section{Keywords}

Acute kidney injury · Citrate $\cdot$ Continuous

hemofiltration - Continuous renal replacement

therapy · Critical care · Dialysis efficiency · Dialysis

machine $\cdot$ Haemodiafiltration

\section{Abstract}

Background/Aims: We assessed how the novel PrisMax continuous renal replacement therapy (CRRT) system performed in a prospective international multicentre setting. We compared this device to its predecessor, the Prismaflex, with regards to multiple treatment parameters. Additionally, we performed a survey, aiming to measure user satisfaction. Methods: Data was prospectively collected from 7 intensive care units (ICU) in 6 countries. The PrisMax device data logs constituted the raw material. Clinical parameters like treatment time, filter life span, downtime, delivered dose and number and type of alarms were recorded. A user questionnaire was sent out to 3 of the participating ICUs. Results: Filter life, downtime, blood pump stops, bag changing time and number of malfunction alarms showed significantly improved values compared to the historic Prismaflex data. The survey showed high scores with regards to user friendliness.
Conclusion: The PrisMax CRRT device is safe and outperformed its' previous generation counterpart in virtually all aspects. Video Journal Club "Cappuccino with Claudio Ronco" at http://www.karger.com/?doi=489213.

(c) 2018 S. Karger AG, Basel

\section{Introduction}

Short, intermittent haemodialysis, as used in critically ill patients during the 1970s was associated with hemodynamic instability, fluctuating levels of electrolytes and suboptimal fluid removal.

The pioneer work from Kramer et al. [1] from Göttingen introduced continuous renal replacement therapy (CRRT). They used continuous arteriovenous hemofiltration, and since then a number of iterations to the therapy have been introduced, improving patient care and safety. Double lumen catheters allow for venovenous hemofiltration, better pumps and improved weighing scales as well as easier priming of the filters are some aspects. In

Marcus Broman and Max Bell contributed equally.

\section{KARGER}

(c) 2018 S. Karger AG, Basel

E-Mail karger@karger.com

www.karger.com/bpu
Marcus Broman

Department of Anesthesiology and Intensive Care

Skåne University Hospital

Getingevägen 4, SE-22185 Lund (Sweden)

E-Mail marcus.broman@skane.se 
later years, introduction of regional anticoagulation, in particular citrate anticoagulation [2,3] has improved filter life span and decreased heparin-associated risks [2].

As the current generation of CRRT devices are getting replaced, the critical care and nephrology community need to evaluate how this latest technology affects their workload and their patients. Will fluid balance be even more precisely handled? Can CRRT downtime be minimized? Are the machines mobile? Can alarm fatigue be addressed? Can user interfaces be improved, leading to decreased nursing workload and improved patient safety?

Recent releases from manufacturers of CRRT devices have added a number of innovations to the broader market. Typically, these innovations focus on expanding the types of purification therapies that can be performed via a single monitor, or attempt to find ways to reduce the complexity or time spent by the user in delivering target prescriptions. Some specific features include algorithms that can track treatment downtime and increase pump speeds to achieve fluid removal targets, simplify management of regional citrate anticoagulation, or simplify fluid handling on input/output of solutions to minimize burden on users.

PrisMax $^{\circledR}$ (Baxter Healthcare Corporation, Chicago, IL, USA) is the latest generation CRRT device aiming to enhance ease of use, renal replacement therapy accuracy, dose delivery and safety.

The present prospective multicentre international pilot study provides a description of the outlines of the PrisMax system and reports how this novel CRRT device performed with regards to prescription and delivery parameters, treatment accuracy and how the user interface was perceived. This study was performed in 6 countries and 7 intensive care units (ICU).

\section{Methods}

The local Ethics Committee approved the study and due to the observational design need for informed consent was waived. Prior to commencing the run-in of this novel piece of technology, a decision was made to use the PrisMax data for the purposes of research.

\section{Study Population}

This prospective observational study was performed in 7 ICUs (Kings College and West Suffolk [UK] 41 filters, CHU Bordeaux [FR] 49 filters, Skåne University Hospital Lund and Malmö sites [SW] 73 filters, Asklepios [DE] 35 filters, Vicenza St Bortolo [IT] 23 filters and The Alfred [AUS] 84 filters) in 6 countries. In total, 305 filters were run. Moreover, historical data from 4,247 filters from the same 7 centres was used as comparison for certain data points.

A Multicentre Pilot Study of the PrisMax CRRT System

\section{CRRT Training}

Nursing staff and physicians underwent an educational program, which included an online tutorial as well as hands-on supervision. PrisMax support was further available 24/7 during the course of the study.

\section{Device Settings}

Most uses of the PrisMax system utilized the ST-150 filter, and regional citrate anticoagulation was used in 188/305 (64.9\%), heparin in 35/305 (11.5\%) and nothing in 72/305 (23.6\%) filters. Patients were treated with continuous venovenous haemodiafiltration (CVVHDF) 236, hemofiltration (CVVH) 61, haemodialysis (CVVHD) 7 and slow continuous ultrafiltration 1 modes. The effluent flow used for all filters were (mean \pm SD) $34.11 \pm 13.42 \mathrm{~mL} /$ $\mathrm{kg} / \mathrm{h}$.

\section{Treatment Data}

The PrisMax device records ongoing data during treatment and the created data logs constituted the raw material. Logical clinical parameters such as treatment time, filter life span, downtime, delivered treatment dose and number and type of alarms were recorded. The PrisMax system data-logs were extracted for statistical analysis.

\section{User Friendliness}

A questionnaire was carried out on a total of 24 experienced nurses in 3 centres: Lund, Sweden (6 nurses), Vicenza, Italy (8 nurses) and Bordeaux, France (10 nurses). In order to qualify to participate in the survey, the nurse had to carry out at least 5 treatments with the new PrisMax model.

The nurses were asked to score (zero = very bad to ten $=$ excellent) the following functions: priming, running, stopping, machine interface, alarm sensitivity, noise during treatment, overall self-instructiveness and mobility of the PrisMax device. The nurse had also to choose one to 3 best and one to 3 worst functions, or to leave the choice blank.

\section{Time-Saving Calculation of Autodrain Function}

Every CRRT prescription creates an effluent bag burden for the nurse proportional to the fluid outflows. The Auto Effluent Accessory is thought to lessen this burden by removing the need of effluent bags.

By measuring the effluent volume from the device, which would have been caught by the effluent bags, if not automatically sent to the drain, and converting it to number of effluent bags by dividing this volume by $5 \mathrm{~L}$, the number of effluent bag changes was obtained. This was then converted to time saving by multiplying with the average time of $123 \mathrm{~s}$ for 1 effluent bag change. To ensure time savings were not overestimated, any downtime or pump stops due to other bag changes, alarms, and pauses from the user, were removed.

Formula 1: ([Average Effluent Flow Rate * (Treatment Duration - Downtime) $] / 5 \mathrm{~L}) *$ (Average Expected Time to Change an Effluent Bag)

\section{Statistical Analysis}

Data was analysed by SAS 9.4; (SAS Institute Inc., Cary, NC, USA) and Excel (Microsoft, Richmond, VA, USA). Descriptive data is expressed in means \pm SD for normally distributed data and median and lower, upper quartiles for non-normally distributed data. Wilcoxon Rank Sum test was used for the comparisons between the novel to the previous Prismaflex system. 


\section{Results}

\section{The PrisMax System}

Figures 1 and 2 show an oversight of the new PrisMax device (Baxter Healthcare Corporation, Chicago, IL, USA) with several new key functions compared to its predecessor Prismaflex, among others the enhanced colour touch screen, the overall quieter machinery and an optional autodrain tubing that removes the need of the waste bag as shown in Figure 3.

\section{Comparison of PrisMax to the Prismaflex Device}

Historic data from the older Prismaflex CRRT device were compared to values from the new PrisMax system generated during the pilot runs. Key functions such as filter life span, total downtime per filter, blood pump stops, general bag changing time, number of informational as well as malfunction alarms showed significantly improved values compared to the historic Prismaflex data as shown in Table 1.

\section{PrisMax Alarms Profile}

There are different types of alarms, ranging from indication of malfunction to informative suggestions. These alarms have 3 levels of priority: high, medium and low. In total, 35,732 alarms occurred during the PrisMax runs.

The 10 most occurring alarms covered two-thirds of all alarms. In Table 2, the 10 most common alarms of any type are presented. These are mostly low priority alarms, where the PrisMax system communicates with the operator running the treatment.

The transpired alarm profiles ranked by priority are presented in Table 3. Serious alarms were uncommon, only making up $3.9 \%$ of all alarms.

\section{PrisMax Autodrain Function and Time Saving}

By using the formula, see the Methods section (Formula 1), we estimated the total time saved by the autodrain function to $31.2 \pm 10.8$ min (mean \pm SD) or $31.2,27.2$ and 36.6 min (median Q1, Q3) per $24 \mathrm{~h}$ treatment time. The mean time of $123 \mathrm{~s}$ for a bag change was used in the calculation. The autodrain function was used in 126 of 304 filters in our cohort.

\section{Down Time Compensation}

The system has an in-built algorithm that tracks downtime up to a total of 10 min running time. It will catch up and increase effluent flow to $2 \mathrm{~mL} / \mathrm{kg} / \mathrm{h}$ or to $20 \%$ of prescribed filtration rate target, whichever is less, in order to compensate. The algorithm is enabled by default but can be switched off.

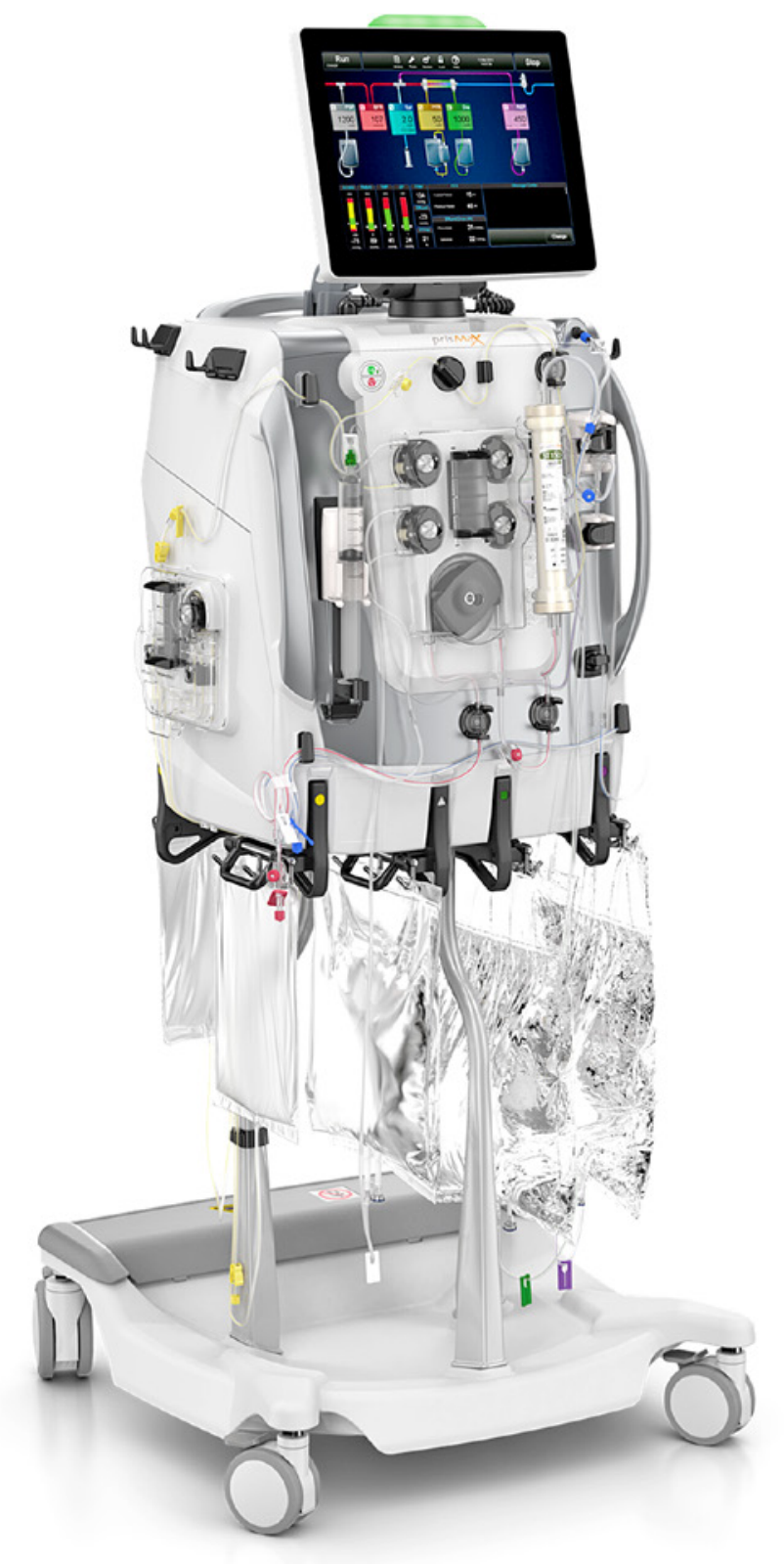

Fig. 1. Overview of the new PrisMax machine layout. It includes a movable multicolour touchscreen with both numerical and visual overview of the selected operations. Note that the effluent bag is replaced by an optional continuous autodrain system. The machine also has a new large 4-wheel carriage, making it stable and mobile during ongoing treatment.

\section{User Friendliness of PrisMax System}

As detailed in the Methods section, a survey was carried out in 3 centres on 24 experienced nurses who had undergone an education package in the new PrisMax system and carried out at least 5 treatment sessions. Tables 


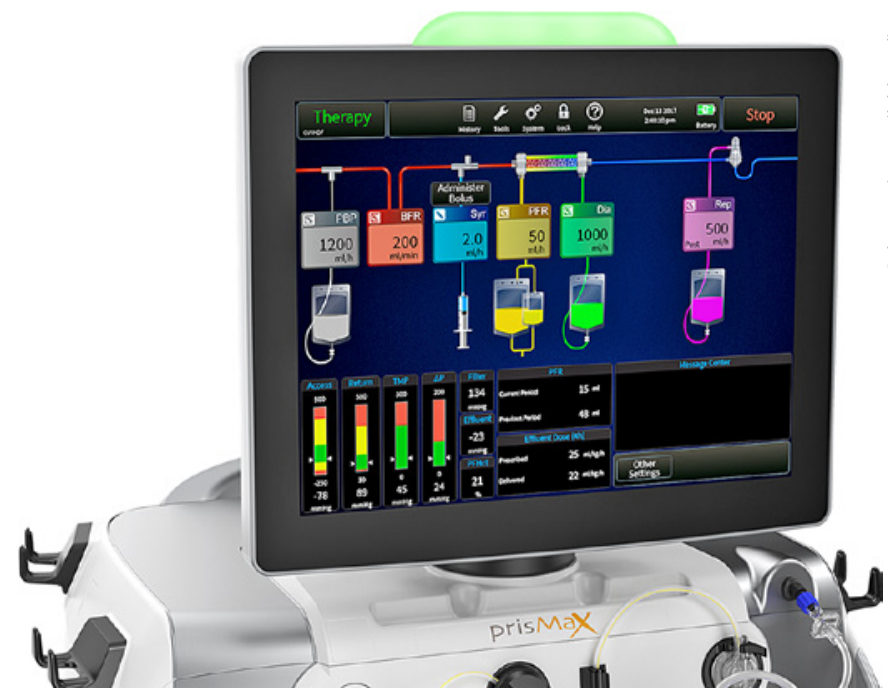

Fig. 2. The running mode screen on PrisMax. Numerical data is mixed with a visual overview of the treatment status, for instance, the content status of the bags. Smooth colouring makes the view clear and systematic. ICU staff might not handle the machine every day and therefore, emphasis has been made to create a self-explanatory interface.

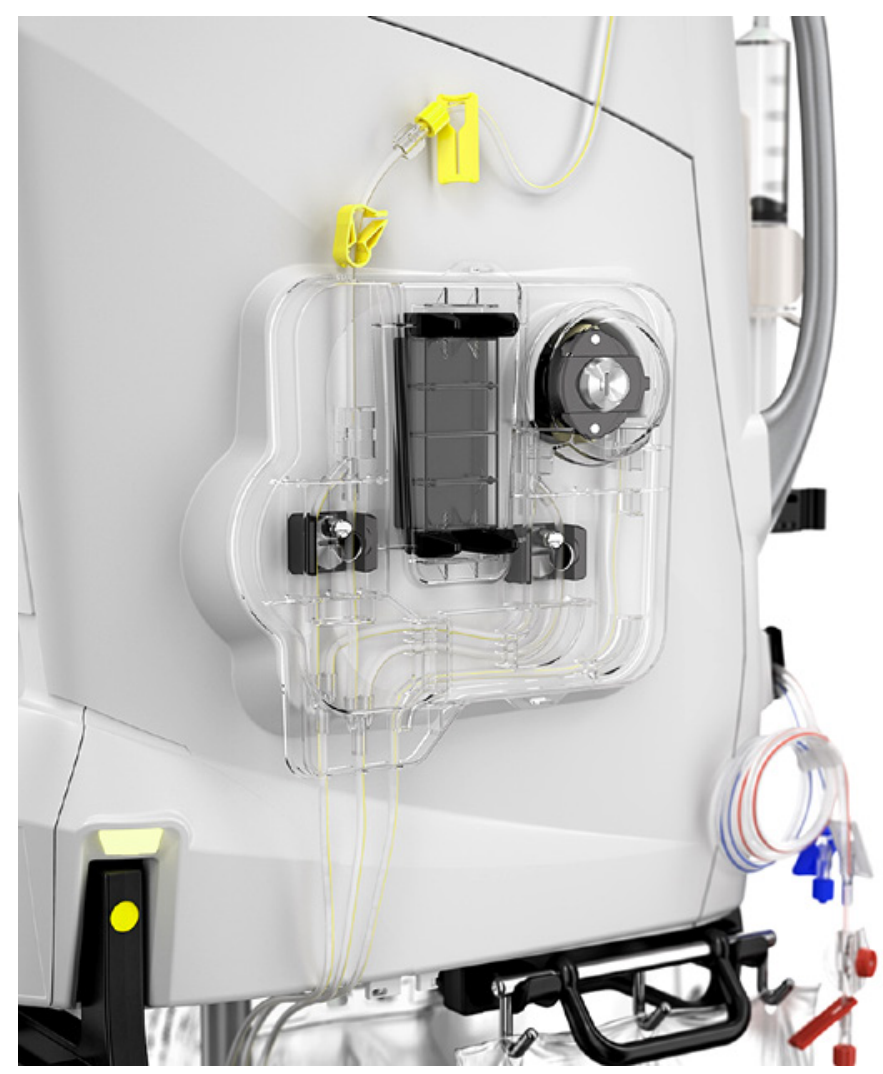

Fig. 3. The optional autodrain function on PrisMax removing the need of an effluent bag. In our multicentre cohort we could show that this function saves time and effort of the staff and yielded high satisfaction scores. It is provided with tubing up to $5 \mathrm{~m}$ (16.4 feet).
$4 \mathrm{a}$ and $4 \mathrm{~b}$ present results from the best/worst functions evaluation. Significantly fewer choices occurred in the worst functions section; 24 choices in total compared to the best functions section; 64 choices in total $(p<0.0001)$. Observe that the nurse could choose $1-3$ best and worst functions, or leave the answer blank.

Figure 4 present data on how PrisMax functionality was scored. All functions received a higher score than the midpoint 5 (scoring range 0 bad to 10 excellent).

\section{Discussion}

In this prospective international multicentre observational study, we conclude that the novel PrisMax system is superior to its predecessor Prismaflex in all parameters tested: from priming duration to filter life span to bag change time. The alarm profile was reasonable with a very low fraction of high-priority alarms indicating malfunction.

The user survey showed that the novel autoeffluent function was popular and we could calculate a significant time saving for the nurses working bedside with the machine. The benefit goes beyond the actual number of minutes saved because the nurse can focus on more important things in a complex patient scenario. In addition, the autodrain system implies a hygiene benefit by removing the handling and emptying of numerous effluent bags.

Discussions on optimal timing of CRRT are still ongoing, as 2 recent randomized controlled trials show conflicting results $[4,5]$. The same can be stated of how renal replacement therapy should be dosed, as none of the large-scale, recent randomized controlled trials adjust for antibiotic losses or removal of low molecular weight heparins in the high-dose arms [6,7]. Other questions remain as well; how do we optimize fluid removal? How do we minimize therapy downtime? These topics interact in many ways with the smartness and user friendliness of the CRRT device. Therefore, it is of utmost importance to create a connection between system developers and clinical users, and even researchers in this field, in order to exchange knowledge. Only this approach will help create the best CRRT systems.

With the introduction of a new line of devices from many manufacturers, we may now have improved safety, reliability and user friendliness, helping intensivists and nephrologists all over to prescribe CRRT more accurately. The present study showed how very few high-priority alarms were set off during treatment with the PrisMax system. 
Table 1. Comparison of key parameters between the previous Prismaflex system and the novel PrisMax system

\begin{tabular}{llll}
\hline & Prismaflex & PrisMax & $\begin{array}{l}\text { Significance level, } \\
p \text { value }\end{array}$ \\
\hline $\begin{array}{lll}\text { Priming time, min } \\
\text { Mean } \pm \text { SD per filter }\end{array}$ & $\begin{array}{l}35.27 \pm 119.58 \\
n=4,230\end{array}$ & $\begin{array}{l}24.07 \pm 43.03 \\
n=305\end{array}$ & 0.4156 \\
\hline Filter life span, $h$ & $25.76 \pm 22.51$ & $32.12 \pm 29.83$ \\
Mean \pm SD per filter & $n=4,181$ & 0.0007 \\
\hline Blood pump stops, min & $34.12 \pm 254.37$ & $6.56 \pm 9.54$ & \\
Mean \pm SD per filter & $n=4,181$ & $n=305$ & $<0.0001$ \\
\hline Bag time change, min & $1.66 \pm 1.17$ & $0.82 \pm 0.31$ & $<0.0001$ \\
Mean \pm SD per filter & $n=3,640$ & $11.97 \pm 8.64$ & \\
\hline Alarms informational, $n$ & $15.62 \pm 22.64$ & $n=305$ & $<0.0001$ \\
Mean \pm SD per filter & $n=4,181$ & $0.22 \pm 0.70$ & \\
\hline Alarms malfunction, $n$ & $0.90 \pm 2.11$ & $n=305$ & $<0.0001$ \\
Mean \pm SD per filter & $n=4,181$ & & \\
\hline
\end{tabular}

Table 2. Presentation of ten most occurring alarm codes during the pilot runs. All except two are low-priority alarms. The two medium-priority alarms in the table are related to access problems

\begin{tabular}{lll}
\hline $\begin{array}{l}\text { Most occurring alarms } \\
\text { Name }\end{array}$ & $\begin{array}{l}\text { Ten most occurring alarms } \\
\text { Type and action }\end{array}$ & Number and frequency, n/\% \\
\hline $\begin{array}{l}\text { Extreme negative access pressure } \\
\text { not self cleared }\end{array}$ & $\begin{array}{l}\text { Medium } \\
\text { Stop all pumps }\end{array}$ & $4,152 / 11.62$ \\
\hline Effluent bag full & $\begin{array}{l}\text { Low } \\
\text { Stop fluid pumps }\end{array}$ & $3,548 / 10.03$ \\
\hline Extreme negative access pressure & Low & $3,260 / 9.12$ \\
self cleared & Stop all pumps & $3,079 / 8.62$ \\
\hline Preblood bag empty & $\begin{array}{l}\text { Low } \\
\text { Stop fluid pumps }\end{array}$ & $2,235 / 6.25$ \\
\hline Effluent scale open & Low & $1,987 / 5.56$ \\
\hline Dialysis bag empty & Stop fluid pumps & \\
\hline Preblood scale open & Low & $1,817 / 5.09$ \\
& Stop fluid pumps & $1,158 / 3.24$ \\
\hline Dialysate scale open & Low & $1,183 / 3.31$ \\
\hline Sheck access & Low fluid pumps & \\
\hline Liquid level sensor low $*$ & Stop fluid pumps & \\
\hline
\end{tabular}

* In the latest iteration of the PrisMax software, this alarm has been removed. Instead a high sensitivity ultrasound sensor has been added. 
A successful CRRT device must be applicable worldwide, and clinical culture and the way people act and react vary. Consequently, a self-instructive interface is very important. The new interface on the PrisMax system was among the most popular functions in the nurses' survey. The system communicates in sharp scenarios through alarms. Out of more than 35,000 alarms, less than $4 \%$ were of high priority, launching when the system announced a possible threat to patient safety with immediate action demanded from the bedside operator. It is essential that the system can identify when a highpriority alarm should be commenced, but it is also as important that the system will not do it repeatedly without adequate reason due to risk of alarm fatigue. This machine function must be tightly streamlined and take into account how both nurses and physicians interact with bedside technology. The small fraction of high priority alarms shows an accuracy of the alarm profile of the system.

The evolution of CRRT machines has been fast. The first commercial systems were deployed widely in the mid to late 1990s. Early devices, such as the Prisma from Hospal/Gambro and the Diapact from Braun, were embraced because of their ability to perform venovenous procedures safely. This allowed clinicians to take control of the patient's volume and electrolytes without compromising their hemodynamic stability. When higher doses of CRRT were targeted, the first-generation machines lacked capacities both with regard to blood flow and effluent flow rates.

The second-generation CRRT devices, like the PrismaFlex (Gambro/Baxter), the Aquarius (Baxter), the NxStage System One (NxStage Medical) and the Multifiltrate (Fresenius) all had the ability to run higher effluent rates, and some provided off-shelf regional citrate anticoagulation solutions.

The current line of devices, that could be described as the third-generation, like the PrisMax (Baxter) - tested in the present study - the MultiFiltrate Pro (Fresenius), the Omni (Braun) as well as other devices from more manufacturers promise improved performance across the board. Indeed, better scale performance and smoother pump abilities could (at least partly) explain the improved filter lifespan described in the present investigation.

The PrisMax, as well as current-generation competitors, present multiple developments that truly could describe them as full-fledged blood purification devices: carbon dioxide removal, sepsis treatment and specific liver filters are possible or even likely future add-ons. On top

A Multicentre Pilot Study of the PrisMax CRRT System
Table 3. Less than $4 \%$ of all 35,732 occurring alarms during the pilot runs were high-priority alarms

\begin{tabular}{lc}
\hline Alarm priority & Number and frequency, $n / \%$ \\
\hline High & $1,399 / 3.92$ \\
Medium & $8,542 / 23.91$ \\
Low & $22,140 / 61.96$ \\
Informational & $3,651 / 10.22$ \\
\hline
\end{tabular}

Table 4.

a Sixty-four choices for the best function

\begin{tabular}{ll}
\hline PrisMax function & Best function \\
\hline Autoeffluent & $19 / 24$ \\
Interface & $19 / 24$ \\
Better overview & $10 / 24$ \\
New touchscreen & $9 / 24$ \\
Mobility & $7 / 24$ \\
\hline
\end{tabular}

b Twenty-four choices for the worst function

\begin{tabular}{ll}
\hline PrisMax function & Worst function \\
\hline Alarm colour coding & $8 / 24$ \\
Not possible to change modality & \\
$\quad$ during ongoing treatment & $5 / 24$ \\
Mobility & $6 / 24$ \\
Stop procedure & $4 / 24$ \\
Screen message centre & $1 / 24$ \\
\hline
\end{tabular}

of that paediatric filters and ECMO compatibility are expected to be introduced.

As an overall analysis, the Prismax scored better compared to its predecessor because of a more interactive interface making the interplay between the nurses and the machine faster. In a complex clinical scenario, there are many parts involved. Nursing reports such as "the machine required less attention", "it ran quieter", "it was easier to get an understanding in which phase the treatment status was" were prevalent. The impression was also that the machine communicated with its operator by using less high-priority alarms. However, this was a structured testing period that included focused pre-education and 24/7 support, which motivated the team to handle the machine, and this can of course create positive bias. The alarm colour coding was the most occurring negative feedback and the opinions presented were that the coding should be made more effective, so that a certain colour code gives an instant understanding of 


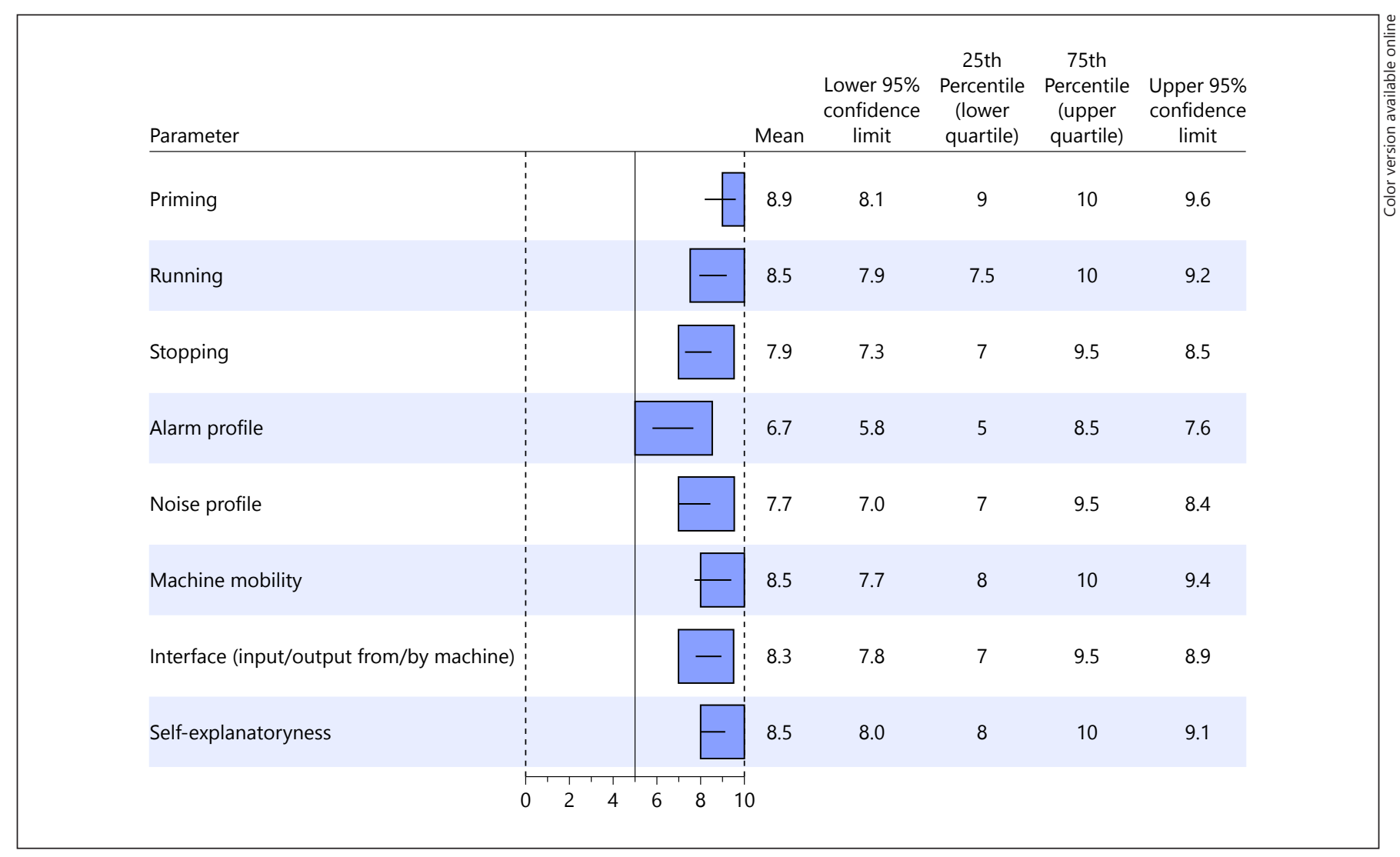

Fig. 4. Nursing survey results.

which kind of alarm is in question. This is a good example of a situation where clinical experts must meet machine developers.

The present study has strengths and weaknesses. The prospective design and the fact that a new CRRT device was simultaneously evaluated in 6 countries are quite unique aspects and allow for generalizability. The fact that we could extricate data logs from the device and not rely on paper-based nursing reports is beneficial. Moreover, we had the ability to compare raw data from the novel device with its predecessor. This gives our study findings a comparator usually not available when new technology is introduced into health care systems. Lastly, our nursing survey, evaluating how bedside users actually think and feel about the device, speaks volumes. Weaknesses exist. The treatments are not uniform in all aspects; for instance, $64.9 \%$ of the treatments in the Prismax group had regional citrate anticoagulation compared to $25.1 \%$ in the historic control group. We would have liked to have even more countries and centres involved, especially from North America and
Asia. More patients and filters would obviously have increased power and generalizability. Also, it would have helped if nursing input was received from all participating hospitals.

In conclusion, this report shows that the new PrisMax CRRT device outperformed its previous generation counterpart in virtually all aspects.

\section{Acknowledgements}

The authors thank all the participating nurses and physicians from all centres. We want to extend our gratitude to Baxter for the support extended by them during this pilot study.

\section{Disclosure Statement}

All authors declare that they have had multiple exchanges with the manufacturer of the PrisMax device, Baxter Healthcare. No financial support pertaining to this study has been awarded, nor has the manufacturer had any influence over the study design or the drafting of the manuscript. 


\section{References}

1 Kramer P, Wigger W, Rieger J, Matthaei D, Scheler F: [Arteriovenous haemofiltration: a new and simple method for treatment of overhydrated patients resistant to diuretics]. Klin Wochenschr 1977;55:1121-1122.

-2 Oudemans-van Straaten HM, Kellum JA, Bellomo R: Clinical review: anticoagulation for continuous renal replacement therapy - heparin or citrate? Crit Care 2011;15:202.

-3 Tolwani AJ, Prendergast MB, Speer RR, Stofan BS, Wille KM: A practical citrate anticoagulation continuous venovenous hemodiafiltration protocol for metabolic control and high solute clearance. Clin J Am Soc Nephrol 2006;1:79-87.
Gaudry S, Hajage D, Schortgen F, MartinLefevre L, Pons B, Boulet E, Boyer A, Chevrel G, Lerolle N, Carpentier D, de Prost N, Lautrette A, Bretagnol A, Mayaux J, Nseir S, Megarbane B, Thirion M, Forel JM, Maizel J, Yonis H, Markowicz P, Thiery G, Tubach F, Ricard JD, Dreyfuss D; AKIKI Study Group: Initiation strategies for renal-replacement therapy in the intensive care unit. $\mathrm{N}$ Engl J Med 2016;375:122-133.

Zarbock A, Kellum JA, Schmidt C, Van Aken $\mathrm{H}$, Wempe C, Pavenstadt H, Boanta A, Gerss J, Meersch M: Effect of early vs delayed initiation of renal replacement therapy on mortality in critically ill patients with acute kidney injury: the ELAIN randomized clinical trial. JAMA 2016;315:2190-2199.
Investigators RRTS, Bellomo R, Cass A, Cole L, Finfer S, Gallagher M, Lo S, McArthur C, McGuinness S, Myburgh J, Norton R, Scheinkestel C, Su S: Intensity of continuous renal-replacement therapy in critically ill patients. N Engl J Med 2009;361:16271638.

Network VNARFT, Palevsky PM, Zhang JH, O'Connor TZ, Chertow GM, Crowley ST, Choudhury D, Finkel K, Kellum JA, Paganini E, Schein RM, Smith MW, Swanson KM, Thompson BT, Vijayan A, Watnick S, Star RA, Peduzzi P: Intensity of renal support in critically ill patients with acute kidney injury. N Engl J Med 2008;359:7-20. 